Article

\title{
A New No Equilibrium Fractional Order Chaotic System, Dynamical Investigation, Synchronization, and Its Digital Implementation
}

\author{
Zain-Aldeen S. A. Rahman ${ }^{1,2} \mathbb{D}$, Basil H. Jasim ${ }^{2} \mathbb{D}$, Yasir I. A. Al-Yasir ${ }^{3, * \mathbb{D}}$, Raed A. Abd-Alhameed ${ }^{3} \mathbb{D}$ \\ and Bilal Naji Alhasnawi ${ }^{2}$ D \\ 1 Department of Electrical Techniques Institute/Qurna, Southern Technical University, Basra 61016, Iraq; \\ as.zain9391@stu.edu.iq \\ 2 Department of Electrical Engineering, College of Engineering, University of Basrah, Basra 61004, Iraq; \\ basil.jasim@uobasrah.edu.iq (B.H.J.); bilalnaji11@yahoo.com (B.N.A.) \\ 3 Biomedical and Electronics Engineering, Faculty of Engineering and Informatics, University of Bradford, \\ Bradford BD7 1DP, UK; R.A.A.Abd@bradford.ac.uk \\ * Correspondence: y.i.a.al-yasir@bradford.ac.uk; Tel.: +44-127-423-8047
}

Citation: Rahman, Z.-A.S.A.; Jasim, B.H.; Al-Yasir, Y.I.A.; Abd-Alhameed, R.A.; Alhasnawi, B.N. A New No Equilibrium Fractional Order Chaotic System, Dynamical Investigation, Synchronization, and Its Digital Implementation. Inventions 2021, 6, 49. https://doi.org/10.3390/ inventions 6030049

Academic Editors: Francisco

Manzano Agugliaro and Esther Salmerón-Manzano

Received: 13 June 2021

Accepted: 30 June 2021

Published: 6 July 2021

Publisher's Note: MDPI stays neutral with regard to jurisdictional claims in published maps and institutional affiliations.

Copyright: (c) 2021 by the authors. Licensee MDPI, Basel, Switzerland. This article is an open access article distributed under the terms and conditions of the Creative Commons Attribution (CC BY) license (https:// creativecommons.org/licenses/by/ $4.0 /)$.

\begin{abstract}
In this paper, a new fractional order chaotic system without equilibrium is proposed, analytically and numerically investigated, and numerically and experimentally tested. The analytical and numerical investigations were used to describe the system's dynamical behaviors including the system equilibria, the chaotic attractors, the bifurcation diagrams, and the Lyapunov exponents. Based on the obtained dynamical behaviors, the system can excite hidden chaotic attractors since it has no equilibrium. Then, a synchronization mechanism based on the adaptive control theory was developed between two identical new systems (master and slave). The adaptive control laws are derived based on synchronization error dynamics of the state variables for the master and slave. Consequently, the update laws of the slave parameters are obtained, where the slave parameters are assumed to be uncertain and are estimated corresponding to the master parameters by the synchronization process. Furthermore, Arduino Due boards were used to implement the proposed system in order to demonstrate its practicality in real-world applications. The simulation experimental results were obtained by MATLAB and the Arduino Due boards, respectively, with a good consistency between the simulation results and the experimental results, indicating that the new fractional order chaotic system is capable of being employed in real-world applications.
\end{abstract}

Keywords: fractional order; dynamics; chaotic; system; synchronization; arduino due

\section{Introduction}

Research on chaotic systems has had a significant practical effect since Lorenz established chaos theory in 1963 [1]. Over the last few decades, nonlinear phenomena in chaos have been widely employed in engineering, science, and applied mathematics [2-4]. Chaos systems with hidden attractors have been the focus of recent research. Self-excited attractors and hidden attractors have been classed as chaotic attractors in dynamical systems since the seminal article by Leonov et al. was investigated [5]. The unstable equilibrium points (system equilibria) are responsible for exciting the basin of attraction in the self-excited chaotic [6]. An attractor, on the other hand, is said to be hidden if its basin of attraction does not intersect with any of the small neighborhoods of the unstable equilibrium [7]. Hidden chaotic attractors are chaotic attractors in dynamical systems with stable equilibria, no equilibrium, and surfaces or lines of equilibria [8].

Chaotic complex systems with hidden attractors are vital in a wide range of scientific and engineering fields, such as bridge wings design [9], induction motors for drilling [10], chemical reactors systems [11], aircraft control systems [12], memristive circuits [13], encryp- 
tion [14], PLL systems [15], weather prediction systems [16], and secure communication schemes [17].

Hidden attractors research in the past has primarily concentrated on integer-order dynamical systems. There have been several studies on complicated chaotic systems with hidden chaotic attractors, such as in [18-23]. The fractional order derivative and fraction order integration calculus have recently received a lot of attention, owing to the fractional calculus providing more accurate models than the integer order [24].

Fractional calculus can be thought of as an expansion of traditional calculus as a branch of mathematical analysis. Due to its possible applications in a variety of domains, the study of fractional calculus has recently received a lot of attention [25]. Fractional calculus can describe many systems in transdisciplinary domains. Furthermore, the fractional-order model can provide an explicit description of the physical process and provide additional insight [26]. Fractional calculus can be used in control, bio-engineering, oscillators, analog filters, circuit theory, image processing encryption systems, and chemistry [27]. Fractional order chaotic models have a more complex dynamical behavior than integer models (since they include the fractional order parameter as well as the original system characteristics); as a result, they are important in secure communications systems [28,29].

The synchronization technique of chaos is based on the principle that two chaotic systems may develop on different attractors, but when synchronized, they begin on different attractors and later follow a single trajectory. When the trajectories of two systems are matched, this synchronization is achieved between these two systems [30]. The control and synchronization techniques of the fractional order chaotic systems can be considered the fundamental challenge of using these systems in many applications as robotics, cryptography, mechanical, and secure communication applications [31,32]. To control and synchronize the fractional-order chaotic systems, a variety of control and synchronization techniques have been developed, such as active control [33], sliding mode control [34], adaptive control [35], passive control [36], and impulsive control [37].

We suggested a new 3D fractional order chaotic system with no equilibrium in this paper, so it can excite hidden chaotic attractors. The system dynamical behaviors including the system equilibria, the chaotic attractors, the bifurcation diagrams, and the Lyapunov exponents were analytically and numerically investigated. Then, two identical new systems, one working as the master (drive) and the other as the slave (response), were used to develop a synchronization mechanism based on adaptive control theory.

Based on Lyapunov's stability theory, the adaptive control laws responsible for tracking and aligning the slave state variables (slave trajectories) with the equivalent trajectories in the master side. Consequently, the update laws for updating the uncertain slave parameters were obtained. By this scenario, the slave was well synchronized with the master. The MATLAB was used to verify our work, testing, and results. Additionally, Arduino Due boards were used to implement a workable hardware electronic circuit for the new system.

The remainder of this paper is organized as follows: the basic mathematical background of the fractional order systems is introduced in Section 2. Section 3 introduces a new fractional order chaotic system and determines its chaotic attractors classes, as well as the system equilibria. In Section 4, the suggested system's dynamical behavior properties are investigated using Lyapunov exponents and bifurcation diagrams. In Section 5, we establish a synchronization approach between two identical new systems, using Lyapunov stability to drive the adaptive control laws and the update laws to achieve the synchronization mechanism and estimate the uncertain slave parameters, respectively. In Section 6, we use the Arduino Due boards to implement the real electronic circuit for the suggested system. In Section 7, we conclude this paper.

\section{Fundamentals of Fractional Order Systems}

Integer-order calculus is mathematically extended to fractional calculus. While it offers the advantages of integer order calculus, it also has its own logic and laws. In the definitions 
of fractional calculus, the Caputo, Riemann-Liouville (RL), and Grunwald-Letnikov (GL) concepts are commonly used [38].

The Gamma function noted by $\Gamma($.$) , which is defined in Equation (1), is the basic$ function used in fractional order calculus [39]:

$$
\Gamma(n)=\int_{0}^{+\infty} \mathrm{e}^{-t} t^{n-1} d t ; n>0
$$

where, $\Gamma(1)=1, \Gamma(0)=+\infty$.

The fractional order calculus introduced by Caputo is stated as follows in Equation (2) [40]:

$$
t_{0} D_{t}^{q} f(t)=\left\{\begin{array}{cc}
\frac{1}{\Gamma(k-q)} \int_{t_{0}}^{t} \frac{f^{(k)}(\tau)}{(t-\tau)^{q-k+1}} d \tau ; & k-1<q<k \\
\frac{d^{k} f(t)}{d t^{k}} ; & q=k
\end{array}\right.
$$

where $k$ is the first integer number that is greater than or equal to fractional q-order.

Equation (3) provides the fractional integral operator $\left(J^{q}\right)$ established by RiemannLiouville for order $(q \geq 0)$ of function $(f(t))[41]$.

$$
J^{q} f(t)= \begin{cases}\frac{1}{\Gamma(q)} \int_{0}^{t}(t-\tau)^{q-1} f(t) d \tau ; & q<0 \\ f(t) ; & q=0\end{cases}
$$

As shown in Equation (4), Grunwald-Letnikov approaches the fractional derivative [42]:

$$
D^{q} x(t)=f(x, t)=\lim _{h \rightarrow 0} h^{-q}(-1) \sum_{j=0}^{t / h}(-1)\left(\begin{array}{c}
q \\
j
\end{array}\right) x(t-j h)
$$

where, $h$ is the step size.

\section{A New Fractional Order Chaotic Model}

Fractional order chaotic systems are a category of nonlinear systems that, in addition to the fundamental characteristics of integer order chaotic systems, have extra features such as extreme complexity and severe sadness behavior [43]. The following Equation (5) can be used to describe the mathematical model of the new three-dimensional fractional order chaotic system proposed in this paper:

$$
\frac{d^{q} x}{d t^{q}}=a z+x y ; \frac{d^{q} y}{d t^{q}}=b-x^{2} ; \frac{d^{q} z}{d t^{q}}=-c x
$$

where the state variables are $x, y$, and $z$; the positive constant system parameters are $a, b$, and $c$, and $q$ presents the fractional order $(0<q<1)$.

The system in Equation (5) exhibits chaotic behavior throughout a wide range of $a, b$, and $c$ parameter values, as well as fractional order $(q)$. The system parameters for the numerical simulation are $a=0.5, b=1.8, c=8$, and $q=0.99$, with initial conditions $(x(0), y(0), z(0))=(1,1,1)$. In 2017, Roberto Garrappa [44] introduced a method for solving the nonlinear fractional order systems, where the simulation results of system (Equation (5)) are obtained based on Roberto Garrappa's method with step size $(h=0.005)$. As illustrated in Figures 1 and 2, the relevant time series of the system states and phase portraits as projections on various planes are obtained, respectively. 


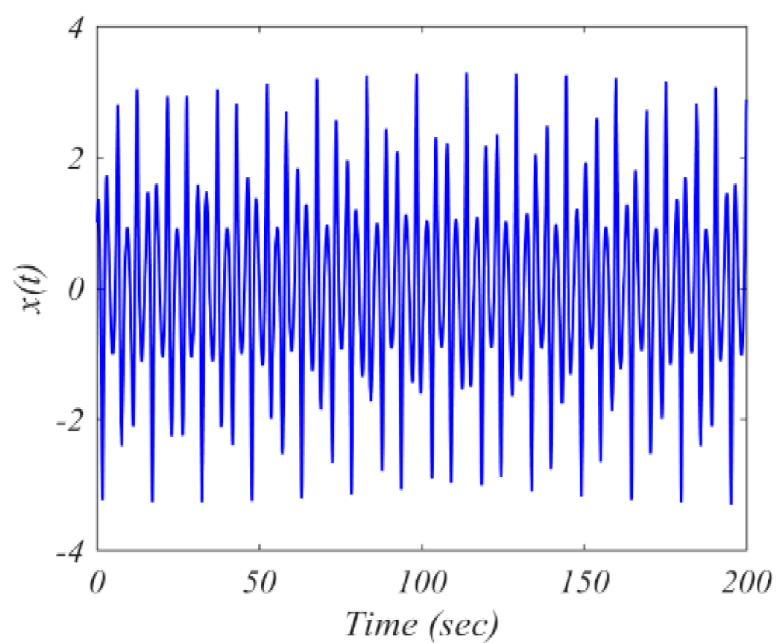

(a)

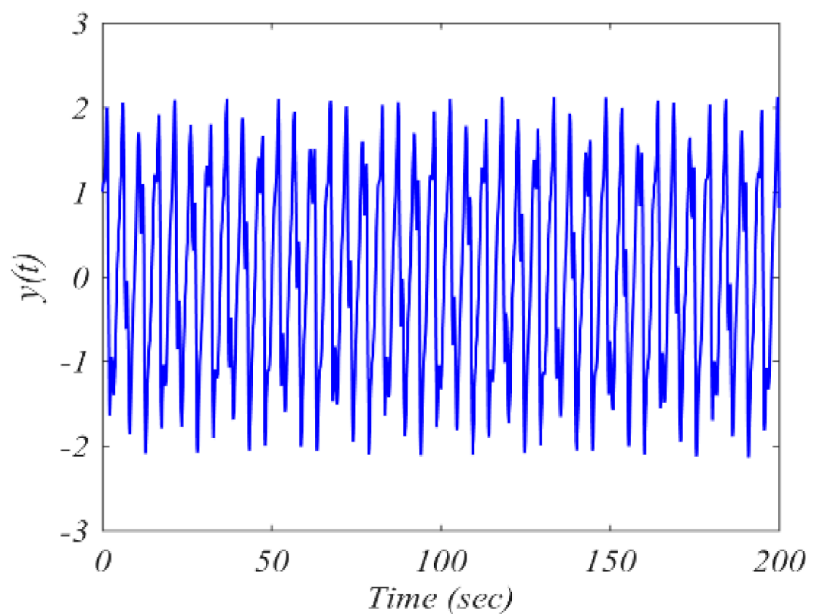

(b)

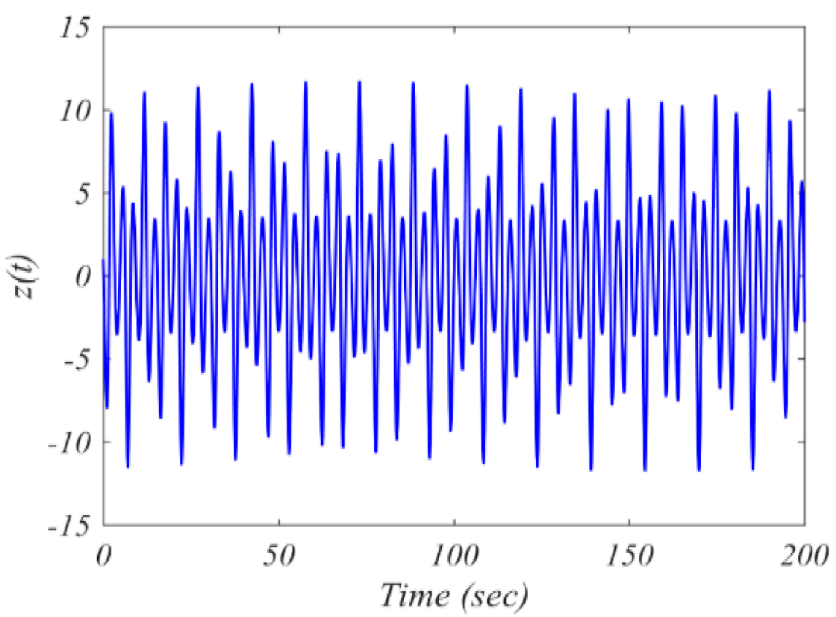

(c)

Figure 1. States time series of proposed system; (a) $x(t) ;(\mathbf{b}) y(t) ;(\mathbf{c}) z(t)$. 


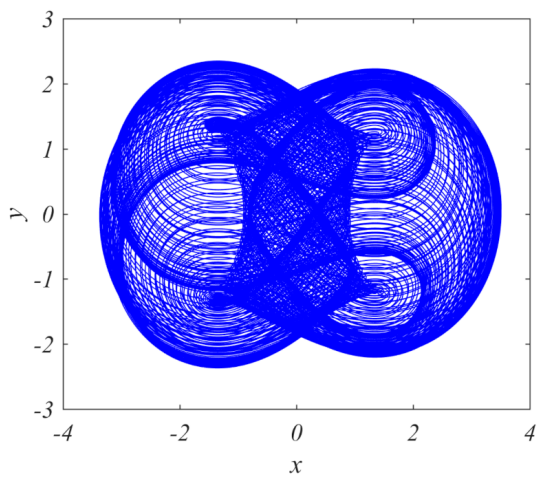

(a)

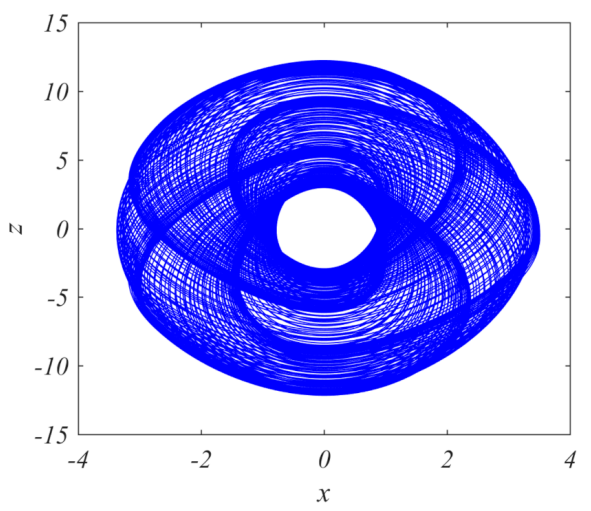

(c)

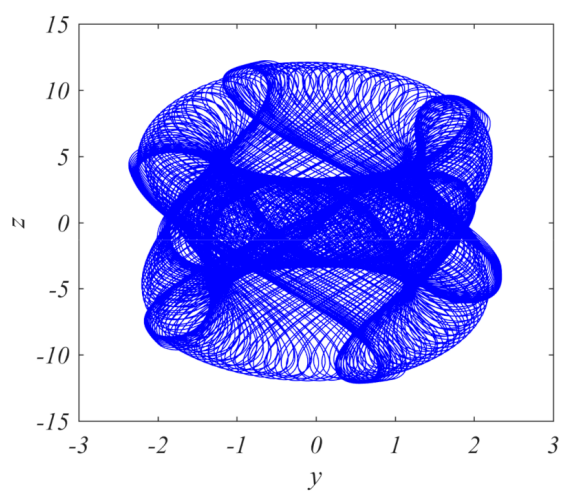

(b)

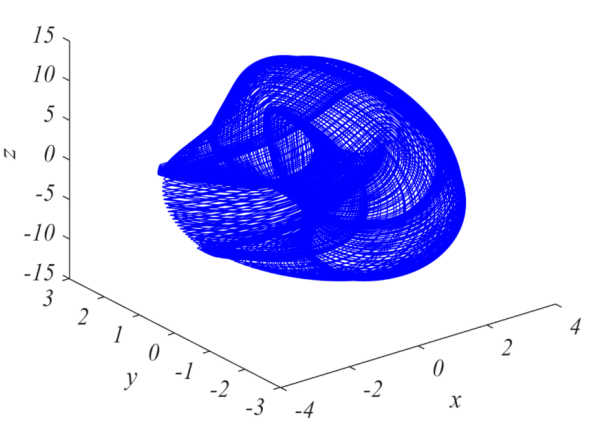

(d)

Figure 2. System (Equation (5)) phase portraits: (a) $x-y$ chaotic attractor; (b) $y$ - $z$ chaotic attractor; (c) $x$ - $z$ chaotic attractor; (d) three-dimensional chaotic attractor.

For determining the equilibrium points (equilibria) of the proposed fractional order chaotic system the state of Equation (5) is equaled by zero as follows in Equation (6):

$$
\frac{d^{q} x}{d t^{q}}=a z+x y=0 ; \frac{d^{q} y}{d t^{q}}=b-x^{2}=0 ; \frac{d^{q} z}{d t^{q}}=-c x=0
$$

As can be noted in Equation (6), there is an inconsistency in Equation (6), where the state variable $x=0$ can be obtained from the third term in Equation (6); however, it is not possible to solve the second term in the same Equation (6) because the constant $b$ cannot equal zero. Therefore, Equation (6) has no solution, which leaves the proposed fractional order chaotic system in Equation (5) without equilibrium points, i.e., the new fractional order chaotic system can excite hidden chaotic attractors.

\section{The System Dynamical Analyses}

Generally, the bifurcation diagrams and Lyapunov exponents are the two main dynamical tools that can be used to investigate the dynamical behaviors of nonlinear chaotic systems [45]. In this section, the bifurcation diagrams and the Lyapunov exponents are numerically investigated by using MATLAB.

\subsection{Bifurcation Diagrams}

The bifurcation diagrams are important means in the nonlinear dynamics and chaos theory. In this work, for exploring the system's dynamical behavior by the bifurcation diagrams, the state variable $y(t)$ of the suggested system is plotted in contradiction with the system parameter $b$, and with respect to the system fractional order $(q)$.

The influence of the system parameter a on the system dynamical behavior are obtained by the bifurcation diagrams as illustrated in Figure 3, where Roberto Garrappa's 
method with step size $(h=0.005)$ and an original program we designed were used for plotting the bifurcation diagrams. The other system parameters are chosen as $a=0.5$ and $c=8$, with initial conditions $(x(0), y(0), z(0))=(1,1,1)$ and fractional order $(q=0.98)$. As can be seen in Figure 3 , the new system exhibits chaotic behavior when the parameter $b>0$.

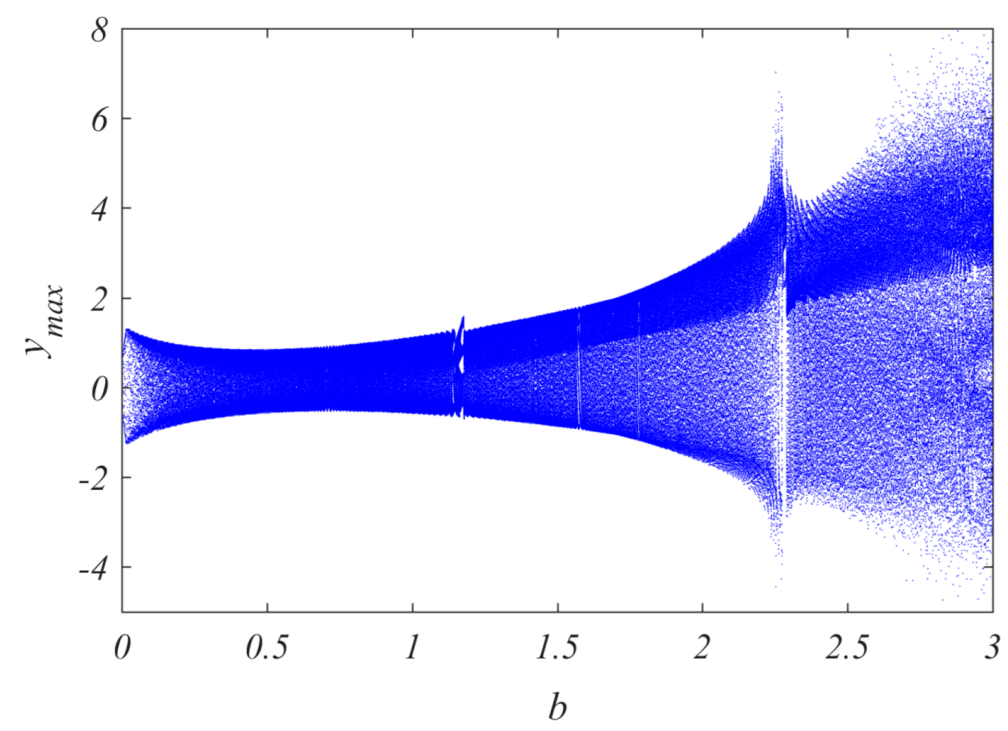

Figure 3. The bifurcation diagram verse system parameter $(b)$.

We then choose the fractional order $(q)$ to be the bifurcation parameter and fixed the parameters $a=0.5, b=1.8$, and $c=8$ with the initial conditions $(1,1,1)$; the dynamical behavior of the system shown in Equation (5) is obtained by the bifurcation diagrams as shown in Figure 4. As can be noted from the bifurcation diagram in Figure 4, the chaotic behavior is excited when the system fractional order $q>0.965$. It can be seen from Figures 3 and 4 , that the system in Equation (5) displays various bifurcation topological patterns.

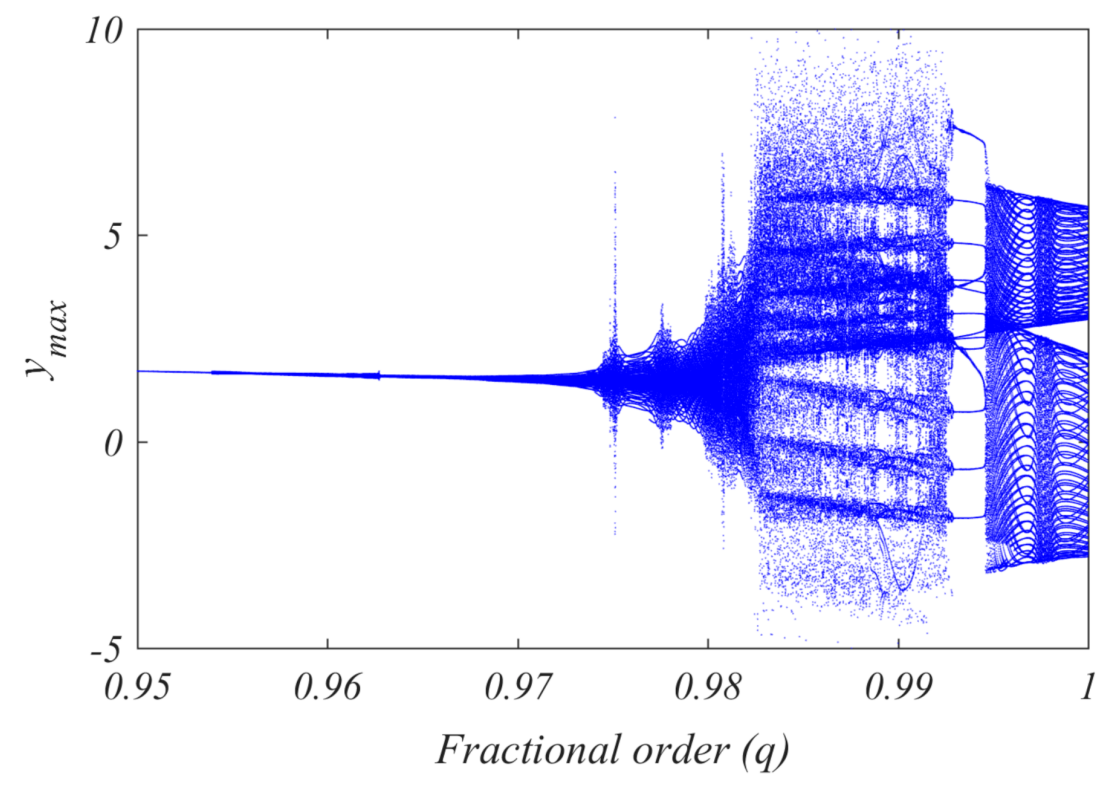

Figure 4. The bifurcation diagram verses the system fractional order $(q)$.

This result shows that the selected system fractional order determines the generation of the hidden chaotic attractor. 


\subsection{Lyapunov Exponents}

The Lyapunov exponents are calculated to strongly indicate that the new system exhibits the chaoticity phenomenon; at least one positive Lyapunov exponent in the nonlinear dynamics system ensures these systems exhibit chaos [46].

Figure 5 demonstrates the Lyapunov exponents with respect to the time (1000 s), where the system parameters are chosen as $a=0.5, b=1.8$, and $c=8$, with initial conditions $(x(0)$, $y(0), z(0))=(1,1,1)$ and fractional order $(q=0.98)$. The consistent Lyapunov exponents are obtained as Le1 $=0.2384$, Le $2=-0.2859$, and Le3 $=-0.3681$. The existence of the positive Lyapunov exponent (Le3) is enough to prove the system in Equation (5) can exhibit the chaos. It should be noted that because Le1 + Le2 + Le $3=-0.3256$, the system in Equation (5) is dissipative, i.e., the new system state trajectories converge into a weird attractor upon conclusion.

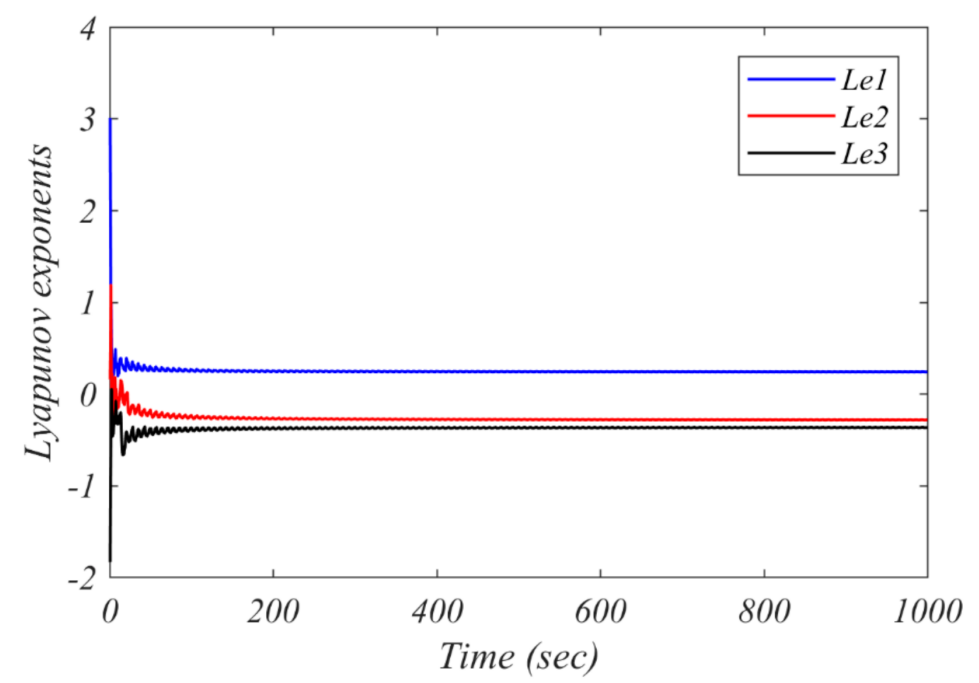

Figure 5. Lyapunov exponents corresponding to time.

In addition, Lyapunov exponents are calculated with respect to changing the fractional order to $q \in[0.95,1]$ as shown in Figure 6. The used system parameters are as mentioned in the first Lyapunov exponents calculations. As can be seen in Figure 6, the Lyapunov exponents are Le $1=0.0287$, Le $2=0.0019$, and Le $3=-0.0017$, which clearly indicate chaotic attractors in the proposed system.

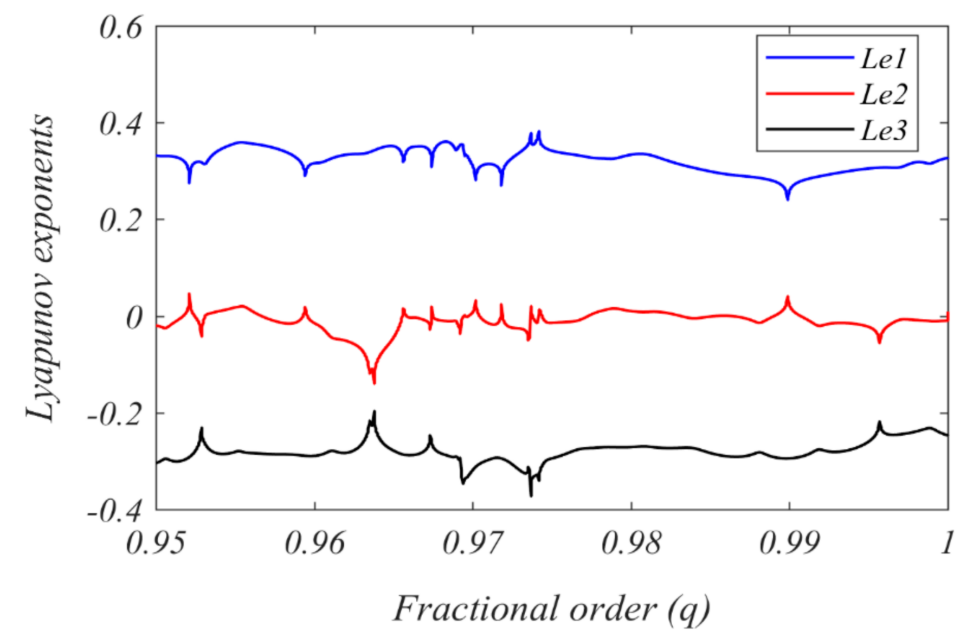

Figure 6. Lyapunov exponents corresponding to the system in Equation (5) fractional order (q). 


\section{Adaptive Synchronization of Two New Fractional Order Chaotic Systems}

Particularly, because the fractional order chaotic complex systems have an extra complexity in dynamical behavior and cannot be described by classical mathematical methods (i.e., deterministic systems), these systems are capable of being widely used in secure communication, image processing, and cryptography systems [47]. Therefore, the fractional order chaotic system synchronization mechanisms have received much attention due to the importance of applications that can be extended into many fields such as physics, engineering, computer science, biology, economics, and brain science. The drive-response (master-slave) form is considered the basic configuration of the chaos synchronization mechanism, where the trajectories of the slave chaotic system must track the trajectories of the master chaotic system. Several methods have been developed to attain the chaos synchronization in fractional-order chaotic systems as mentioned in Section 1.

In this work, we developed an adaptive synchronization technique in order to achieve synchronization between two identical new systems, where the first acts as the master (drive) system and the second acts as the slave (response) system. Based on Lyapunov's theory, the synchronization controllers and the slave parameter estimation laws were derived. The adaptive control laws drive the slave so its trajectories and track the analogues master trajectories, and the slave parameter estimation laws are used for updating the uncertain slave parameters corresponding to the known analogous master parameters.

The adaptive synchronization technique has many benefits, such as good transient performance, rapid dynamics responses, and a robust system for parameter variations and initial conditions.

\subsection{Adaptive Controller Design Process}

In this subsection, we present the design of the adaptive controller for achieving the synchronization between two identical new fractional order chaotic systems. The master and slave state equations are presented by Equations (7) and (8), respectively:

$$
\begin{gathered}
\frac{d^{q} x_{m}}{d t^{q}}=a_{m} z_{m}+x_{m} y_{m} ; \frac{d^{q} y_{m}}{d t^{q}}=b_{m}-x_{m}{ }^{2} ; \frac{d^{q} z}{d t^{q}}=-c_{m} x_{m} \\
\frac{d^{q} x_{s}}{d t^{q}}=a_{s}(t) z_{s}+x_{s} y_{s}+u_{1} ; \frac{d^{q} y_{s}}{d t^{q}}=b_{s}(t)-x_{s}{ }^{2}+u_{2} ; \frac{d^{q} z}{d t^{q}}=-c_{s}(t) x_{s}+u_{3}
\end{gathered}
$$

where, $u_{1}, u_{2}$, and $u_{3}$ are the adaptive synchronization controllers that we want to design; $a_{m}, b_{m}$, and $c_{m}$ are the known master parameters; and $a_{s}(t), b_{s}(t)$, and $c_{s}(t)$ present the uncertain slave parameters that must be estimated. The master-slave synchronization errors can be determined by Equation (9).

$$
e_{x}=x_{s}-x_{m} ; e_{y}=y_{s}-y_{m} ; e_{z}=z_{s}-z_{m}
$$

The dynamic errors are determined as in Equation (10).

$$
\begin{gathered}
\frac{d^{q} e_{x}}{d t^{q}}=a_{2} e_{z}+x_{s} y_{s}-x_{m} y_{m}+z_{m} e_{a}+u_{1} ; \frac{d^{q} e_{y}}{d t^{q}}=e_{b}-x_{s}^{2}+x_{m}^{2}+u_{2} ; \\
\frac{d^{q} e_{z}}{d t^{q}}=c_{2} e_{x}+x_{m} e_{c}+u_{3}
\end{gathered}
$$

where $e_{a}, e_{b}$, and $e_{c}$ are the errors of master-slave parameters and can be determined as in Equation (11).

$$
e_{a}=a_{s}(t)-a_{m} ; \quad e_{b}=b_{s}(t)-b_{m} ; \quad e_{c}=c_{s}(t)-c_{m}
$$

The dynamics of the parameter errors can be calculated by Equation (12).

$$
\dot{e}_{a}=\dot{a}_{s}(t) ; \quad \dot{e}_{b}=\dot{b}_{s}(t) ; \quad \dot{e}_{c}=\dot{c}_{s}(t)
$$

We used the Lyapunov strategy to design the adaptive controllers to verify the adaptive master-slave synchronization mechanism. Consequently, the update laws for estimat- 
ing the uncertain parameters were obtained. Therefore, the quadratic positive Lyapunov functions are used as in Equation (13).

$$
V\left(e_{x}, e_{y}, e_{z}, e_{a}, e_{b}, e_{c}\right)=\frac{1}{2}\left(e_{x}^{2}+e_{y}^{2}+e_{z}^{2}+e_{a}^{2}+e_{b}^{2}+e_{c}^{2}\right)
$$

The results of the Lyapunov function dynamics are obtained as in Equation (14).

$$
\dot{V}=\left(e_{x} \frac{d^{q} e_{x}}{d t^{q}}+e_{y} \frac{d^{q} e_{y}}{d t^{q}}+e_{z} \frac{d^{q} e_{z}}{d t^{q}}+e_{a} \dot{e}_{a}+e_{b} \dot{e}_{b}+e_{c} \dot{e}_{c}\right)
$$

Equations (10)-(12) are substituted in Equation (14) to obtain the following Equation (15).

$$
\begin{aligned}
\dot{V}= & \left(e_{x}\left(a_{2} e_{z}+x_{s} y_{s}-x_{m} y_{m}+z_{m} e_{a}+u_{1}\right)+e_{y}\left(e_{b}-x_{s}^{2}+x_{m}^{2}+u_{2}\right)\right. \\
& +e_{z}\left(c_{2} e_{x}+x_{m} e_{c}+u_{3}\right)+\left(a_{s}(t)-a_{m}\right) \dot{a}_{s}(t)+\left(b_{s}(t)\right. \\
& \left.-b_{m}\right) \dot{b}_{s}(t)+\left(c_{s}(t)-c_{m}\right) \dot{c}_{s}(t)
\end{aligned}
$$

Thus, Equation (15) was used to design the synchronization controllers as in the following Equation (16).

$$
u_{1}=-k_{x} e_{x}-a_{s} e_{z}-y_{s} z_{s}+y_{m} z_{m} ; u_{2}=-k_{y} e_{y}+x_{s}{ }^{2}-x_{m}{ }^{2} ; u_{3}=-k_{z} e_{z}-c_{s} e_{x}
$$

In Equation (16), $k_{x}, k_{y}$, and $k_{z}$ present positive constants, and the uncertain slave system parameters included $\left(a_{s}, b_{s}\right.$, and $\left.c_{s}\right)$ are estimated by updated laws as in the following Equation (17).

$$
\dot{a}_{s}(t)=-z_{m} e_{x} ; \dot{b}_{s}(t)=-e_{y} ; \dot{c}_{s}(t)=-x_{m} e_{z}
$$

Finally, the following Equation (18) is obtained by substituting Equations (16) and (17) in Equation (15).

$$
\dot{V}=-k_{x} e_{x}^{2}-k_{y} e_{y}^{2}-k_{z} y^{2}
$$

As can be seen from Equation (17), there is a negative definite function [48]. As a result, given any initial conditions, the synchronization state errors and the master-slave parameter estimation error converge to zero exponentially with respect to time.

\subsection{Simulation Results}

Numerical studies using the MATLAB platform are used to confirm the efficiency of the suggested synchronization strategy. Table 1 shows the values of the master and slave parameters, fractional orders, and initial conditions utilized to simulate the aforementioned synchronization technique.

Table 1. Master and slave parameters values.

\begin{tabular}{cccc}
\hline \multicolumn{2}{c}{ Master System } & \multicolumn{2}{c}{ Slave System } \\
\hline Parameter & Value & Parameter & Value \\
\hline$a_{m}$ & 0.5 & $a_{s}(t)$ & Estimated \\
$b_{m}$ & 1.8 & $b_{s}(t)$ & Estimated \\
$c_{m}$ & 8 & $c_{m}(t)$ & Estimated \\
fractional order $(q)$ & 0.98 & fractional order $(q)$ & 0.98 \\
$X_{m}(0)$ & 1 & $X_{s}(0)$ & 2 \\
$y_{m}(0)$ & 1 & $y_{s}(0)$ & 0 \\
$z_{m}(0)$ & 1 & $z_{s}(0)$ & -1 \\
\hline
\end{tabular}

The slave trajectories (state variables) effectively follow the master trajectories based on the derived adaptive control laws in Equation (16) as shown in Figure 7. Although initial conditions have different signs and values, the simulation results demonstrate that the master and slave state variables were synchronized in a short time, indicating that the 
developed controller is efficient. In Figure 8, the synchronization errors $\left(e_{x}, e_{y}\right.$, and $\left.e_{z}\right)$ are illustrated. It can be seen that the synchronization errors rapidly (in less than $1.5 \mathrm{~s}$ ) decrease to zero values exponentially. As illustrated in Figure 9, these uncertain slave parameters were appropriately estimated to the corresponding master parameters using the updated laws in Equation (17).

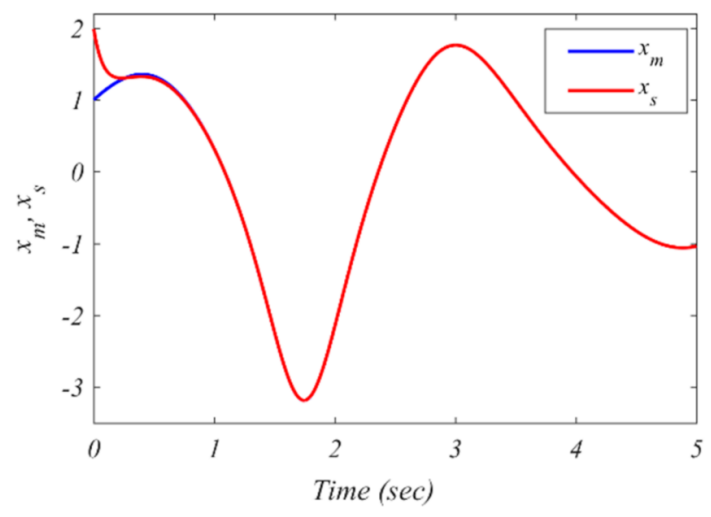

(a)

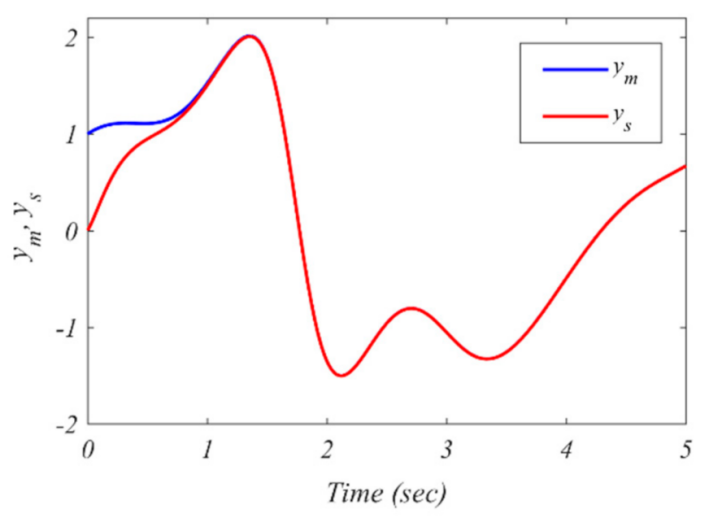

(b)

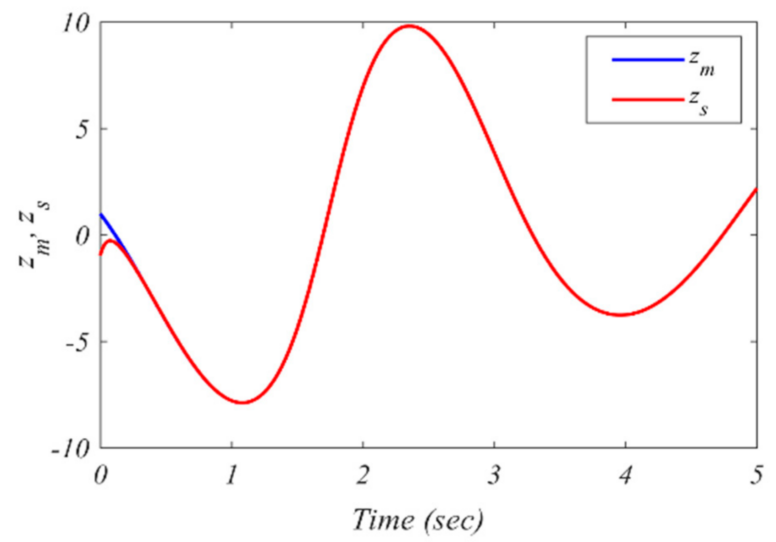

(c)

Figure 7. Tracking the slave trajectories corresponding to master trajectories; (a) $x_{s} \operatorname{track} x_{m} ;(\mathbf{b}) y_{s} \operatorname{track} y_{m} ;(\mathbf{c}) z_{s}$ track $z_{m}$.

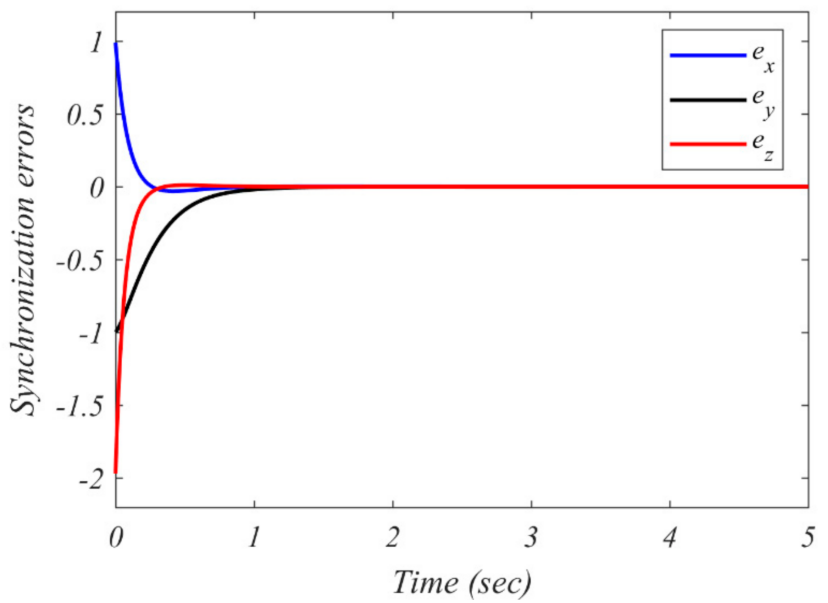

Figure 8. The state variables synchronization errors. 


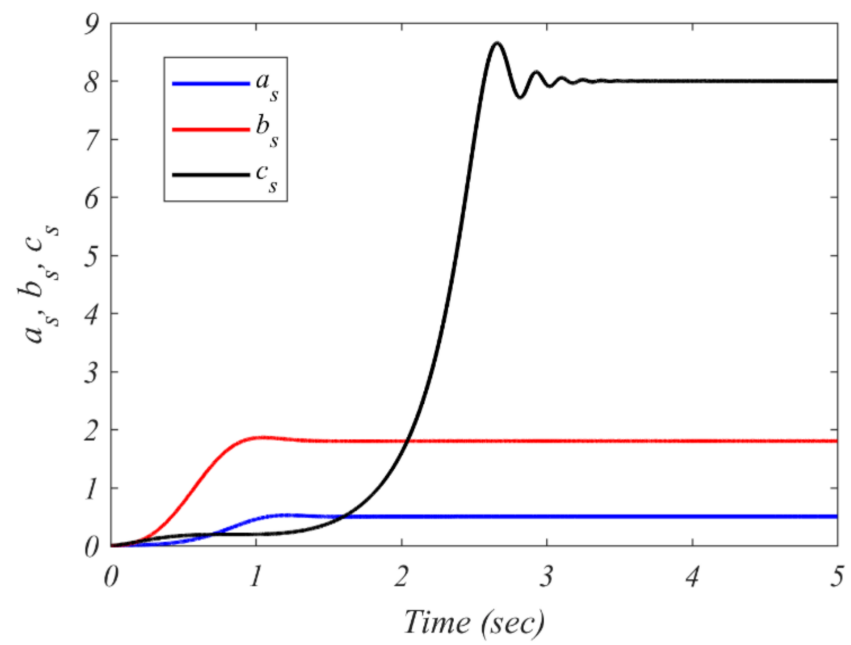

Figure 9. Estimation of uncertain slave parameters corresponding to master trajectories.

As can be seen from Figure 9, that the uncertain slave parameters, $a_{s}$ and $b_{s}$, are rapidly estimated corresponding to the master parameter $a_{m}$ and $b_{m}$, respectively (in less than $1.5 \mathrm{~s}$ ), which is an exact match to the time duration of the synchronization errors to reach zero. On the other hand, the time duration was about $3.5 \mathrm{~s}$ for estimating the third uncertain slave parameter $c_{s}$ corresponding to the master parameter $c_{m}$, where it does not affect the synchronization process. This is because the dynamical behavior of the nonlinear systems is not affected by the same degree of sensitivity for all its parameters as mentioned in [49].

\section{Digital Implementation of New Fractional Order Chaotic System}

The major goal of the hardware is to test the possibility of implementing fractional order chaotic systems so that they can be employed in real-world applications. Fractional order chaotic systems can be implemented in hardware utilizing a variety of embedded devices, such as microcontrollers, Raspberry Pi, FPGAs, and DSPs as well as implementation by analog electronic circuit as in [29]. In this work, we implemented the new three-dimensional system in Equation (5) by using a microcontroller (Arduino Due) based on a discrete method as in [50].

The Arduino Due is a digital board with Atmel SAM3X8E and ARM Cortex-M3 CPU. It consists of an ideal structure for performing complex arithmetic operations. Briefly, it has the following characteristics: 32-bit ARM core microcontroller; $84 \mathrm{MHz}$ clock; 54 digital input/output pins; 12 analog inputs; USB OTG capable connection; 4 UARTs; 2 DAC (digital to analog); power jack 2 TWI; SPI header; and JTAG header. The primary reasons to use this microcontroller are: 12 bit resolution for its two peripherals, DAC0 and DAC1, and its attractive cost advantage compared with the FPGAs or DSPs boards. An Arduino specified programing language similar to $\mathrm{C}++$ language is used for programing the ARM microcontroller by the Arduino IDE through the native USB port (serial port) [51]. The hardware implementation of the new fractional order chaotic system is shown in Figure 10.

The system parameters for the experimental test are $a=0.5, b=1.8, c=8$, and $q=0.99$, with initial conditions $(x(0), y(0)$, and $z(0))=(1,1,1)$. The ADC 0 and DAC1 are used to give the system in Equation (5) phase portraits of chaotic attractors by analog oscilloscope as displayed in Figure 11. In fact, because the microcontroller's digital to analog converters (DAC0 and DAC1) operate between $0.5 \mathrm{~V}$ and $2.7 \mathrm{~V}$, the amplitudes of the simulation results by MATLAB and the experimental results by the Arduino Due will differ for the system's (Equation (5)) state variables (system trajectories). It would be necessary to install an external operational amplification stage in order to achieve the same amplitude values as the computed numerical simulations. Based on the approach that was used for implementing the system (Equation (5)) by the Arduino Due board, there is a level of error of about $\pm 1.56 \%$ compared with the simulation results obtained by MATLAB. 


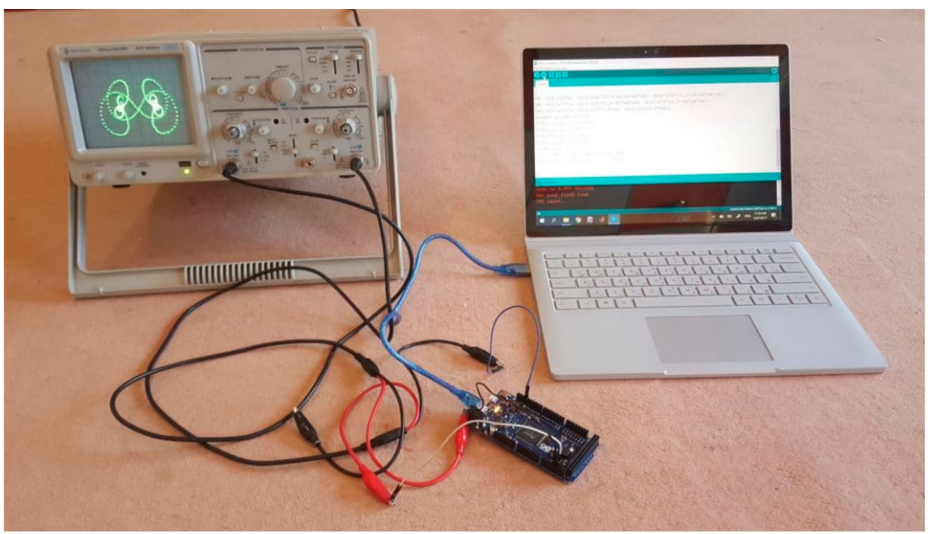

Figure 10. Hardware implementation of the system in Equation (5) based on Arduino Due board.

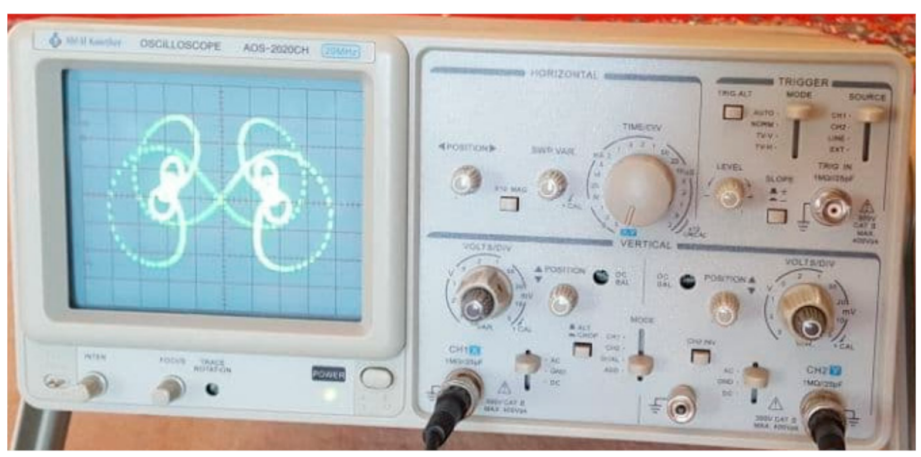

(a)

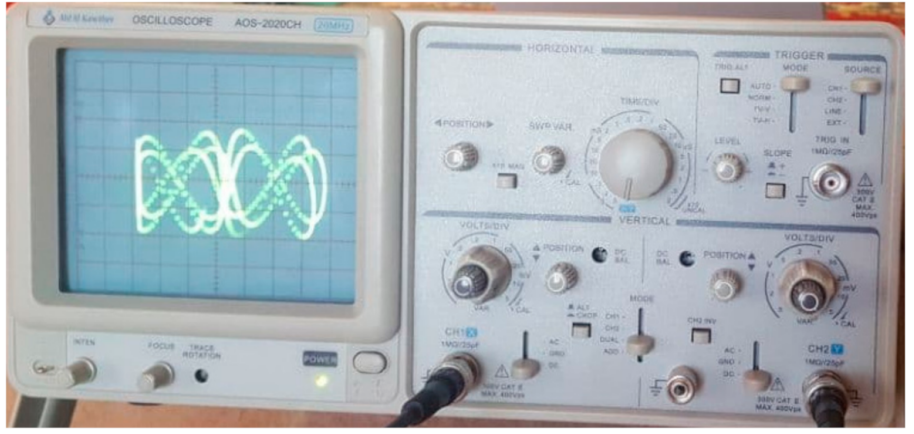

(b)

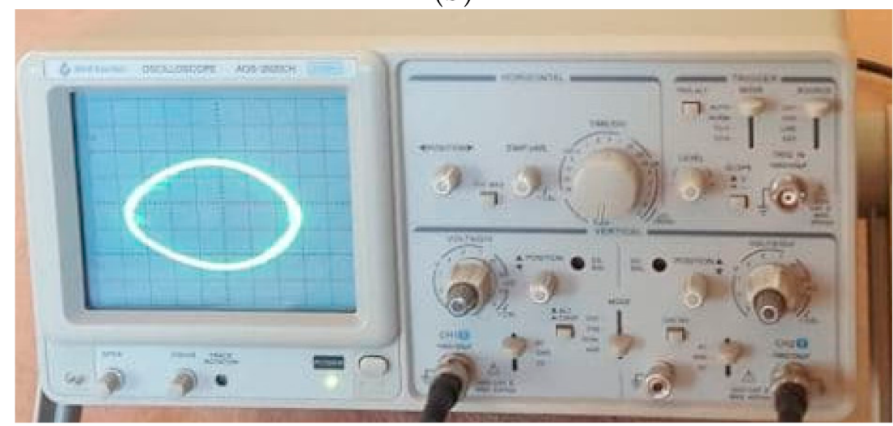

(c)

Figure 11. The experimental results of the proposed system phase portraits: (a) $x-y$ chaotic attractors; (b) $y$ - $z$ chaotic attractors; (c) $x-z$ chaotic attractors.

\section{Conclusions}

A new three-dimensional nonlinear autonomous system with fractional order and chaos exhibition was suggested. The nonlinear dynamical behaviors of this system were analytically and numerically investigated, where these dynamics are the equilibrium points, 
chaotic attractors, bifurcation diagrams, and Lyapunov exponents. Because the system has no equilibrium, the observed dynamics showed that the system can excite hidden chaotic attractors and display extremely complex dynamics. Afterwards, an adaptive synchronization strategy was formulated. This synchronization approach was set up between two identical new fractional order chaotic systems: one serving as the master and the other as the slave. The adaptive control principles responsible for synchronization verification were derived. Furthermore, in order to estimate the unknown slave parameters, the update laws were determined. Finally, to show the feasibility of using the proposed system in real-world applications, the system was implemented with Arduino Due boards. The obtained numerical results by MATLAB simulation are consistent with the experimental results of the hardware implementation, which show the feasibility of the system to be used in real-world applications in various fields. The main advantage of implementation of this system with Arduino Due boards is the low cost of implementation compared with alternative devices such as FPGAs and DSPs, which require large costs.

Author Contributions: Conceptualization, Z.-A.S.A.R. and B.H.J.; methodology, Z.-A.S.A.R.; software, Z.-A.S.A.R. and B.H.J.; validation, Z.-A.S.A.R. and B.H.J.; formal analysis, Z.-A.S.A.R., B.H.J., and Y.I.A.A.-Y.; investigation, Z.-A.S.A.R. and B.H.J..; resources, Z.-A.S.A.R., B.H.J., Y.I.A.A.-Y., R.A.A.-A. and B.N.A.; data curation, Z.-A.S.A.R. and B.H.J.; writing-original draft preparation, Z.-A.S.A.R. and B.H.J.; writing-review and editing, Z.-A.S.A.R., B.H.J., Y.I.A.A.-Y. and B.N.A.; visualization, Z.-A.S.A.R., B.H.J., Y.I.A.A.-Y. and B.N.A.; supervision, B.H.J. and R.A.A.-A. All authors have read and agreed to the published version of the manuscript.

Funding: This research received no external funding.

Institutional Review Board Statement: Not applicable.

Informed Consent Statement: Not applicable.

Conflicts of Interest: The authors declare no conflict of interest.

\section{References}

1. Sordi, A. Chua's oscillator: An introductory approach to chaos theory. Rev. Bras. Ensino Física 2021, 43. [CrossRef]

2. Vaidyanathan, S.; Volos, C. Advances and Applications in Chaotic Systems; Springer: Berlin/Heidelberg, Germany, 2016; Volume 636.

3. Asiain, E.; Garrido, R. Anti-Chaos control of a servo system using nonlinear model reference adaptive control. Chaos Solitons Fractals 2021, 143, 110581. [CrossRef]

4. Cuellar, G.H.; Jiménez-López, E.; Campos-Cantón, E.; Pisarchik, A. An approach to generate deterministic Brownian motion. Commun. Nonlinear Sci. Numer. Simul. 2014, 19, 2740-2746. [CrossRef]

5. Jiang, H.; Liu, Y.; Wei, Z.; Zhang, L. Hidden chaotic attractors in a class of two-dimensional maps. Nonlinear Dyn. 2016, 85, 2719-2727. [CrossRef]

6. Jasim, B.H.; Hassan, K.H.; Omran, K.M. A new 4-D hyperchaotic hidden attractor system: Its dynamics, coexisting attractors, synchronization and microcontroller implementation. Int. J. Electr. Comput. Eng. 2021, 11, 2068-2078. [CrossRef]

7. Volos, C.K.; Jafari, S.; Kengne, J.; Munoz-Pacheco, J.M.; Rajagopal, K. Nonlinear dynamics and entropy of complex systems with hidden and self-excited attractors. Entropy 2019, 21, 370. [CrossRef]

8. Azar, A.T.; Serrano, F.E. Stabilization of Port Hamiltonian Chaotic Systems with Hidden Attractors by Adaptive Terminal Sliding Mode Control. Entropy 2020, 22, 122. [CrossRef]

9. Leonov, G.; Kuznetsov, N.V.; Mokaev, T. Homoclinic orbits, and self-excited and hidden attractors in a Lorenz-like system describing convective fluid motion. Eur. Phys. J. Spéc. Top. 2015, 224, 1421-1458. [CrossRef]

10. Leonov, G.; Kuznetsov, N.V.; Kiseleva, M.; Solovyeva, E.P.; Zaretskiy, A.M. Hidden oscillations in mathematical model of drilling system actuated by induction motor with a wound rotor. Nonlinear Dyn. 2014, 77, 277-288. [CrossRef]

11. Sambas, A.; Vaidyanathan, S.; Bonny, T.; Zhang, S.; Sukono; Hidayat, Y.; Gundara, G.; Mamat, M. Mathematical Model and FPGA Realization of a Multi-Stable Chaotic Dynamical System with a Closed Butterfly-Like Curve of Equilibrium Points. Appl. Sci. 2021, 11, 788. [CrossRef]

12. Andrievsky, B.; Kuznetsov, N.; Leonov, G.; Pogromsky, A. Hidden oscillations in aircraft flight control system with input saturation. IFAC Proc. Vol. 2013, 46, 75-79. [CrossRef]

13. Pham, V.T.; Jafari, S.; Vaidyanathan, S.; Volos, C.; Wang, X. A novel memristive neural network with hidden attractors and its circuitry implementation. Sci. China Ser. E Technol. Sci. 2015, 59, 358-363. [CrossRef]

14. Kiani-B, A.; Fallahi, K.; Pariz, N.; Leung, H. A chaotic secure communication scheme using fractional chaotic systems based on an extended fractional Kalman filter. Commun. Nonlinear Sci. Numer. Simul. 2009, 14, 863-879. [CrossRef] 
15. Kuznetsov, N.V.; Leonov, G.A.; Yuldashev, M.V.; Yuldashev, R.V. Hidden attractors in dynamical models of phase-locked loop circuits: Limitations of simulation in MATLAB and SPICE. Commun. Nonlinear Sci. Numer. Simul. 2017, 51, 39-49. [CrossRef]

16. Vlachas, P.R.; Byeon, W.; Wan, Z.Y.; Sapsis, T.P.; Koumoutsakos, P. Data-driven forecasting of high-dimensional chaotic systems with long short-term memory networks. Proc. R. Soc. A Math. Phys. Eng. Sci. 2018, 474, 20170844. [CrossRef]

17. Velamore, A.A.; Hegde, A.; Khan, A.A.; Deb, S. Dual cascaded Fractional-order Chaotic Synchronization for Secure Communication with Analog Circuit Realisation. In Proceedings of the 2021 IEEE Second International Conference on Control, Measurement and Instrumentation (CMI), Kolkata, India, 8-10 January 2021; IEEE: New York, NY, USA, 2021; pp. 30-35.

18. Pham, V.-T.; Vaidyanathan, S.; Volos, C.K.; Jafari, S. Hidden attractors in a chaotic system with an exponential nonlinear term. Eur. Phys. J. Spéc. Top. 2015, 224, 1507-1517. [CrossRef]

19. Tahir, F.R.; Jafari, S.; Pham, V.-T.; Volos, C.; Wang, X. A Novel No-Equilibrium Chaotic System with Multiwing Butterfly Attractors. Int. J. Bifurc. Chaos 2015, 25. [CrossRef]

20. Pham, V.-T.; Vaidyanathan, S.; Volos, C.; Kapitaniak, T. Nonlinear Dynamical Systems with Self-Excited and Hidden Attractors; Springer: Berlin/Heidelberg, Germany, 2018; Volume 133.

21. Bayani, A.; Rajagopal, K.; Khalaf, A.J.M.; Jafari, S.; Leutcho, G.D.; Kengne, J. Dynamical analysis of a new multistable chaotic system with hidden attractor: Antimonotonicity, coexisting multiple attractors, and offset boosting. Phys. Lett. A 2019, 383, 1450-1456. [CrossRef]

22. Rahman, Z.-A.S.A.; Jassim, B.H.; Al-Yasir, Y.I.A. New Fractional Order Chaotic System: Analysis, Synchronization, and it's Application. Iraqi J. Electr. Electron. Eng. 2021, 17. [CrossRef]

23. Escalante-González, R.J.; Campos, E. Multistable systems with hidden and self-excited scroll attractors generated via piecewise linear systems. Complex. Dyn. Control Appl. Nonlinear Syst. Multistability 2020, 2020. [CrossRef]

24. Ma, C. A Novel Computational Technique for Impulsive Fractional Differential Equations. Symmetry 2019, 11, 216. [CrossRef]

25. Sun, H.; Zhang, Y.; Baleanu, D.; Chen, W.; Chen, Y. A new collection of real world applications of fractional calculus in science and engineering. Commun. Nonlinear Sci. Numer. Simul. 2018, 64, 213-231. [CrossRef]

26. Boubaker, O.; Jafari, S. Recent Advances in Chaotic Systems and Synchronization: From Theory to Real World Applications; Academic Press: Amsterdam, The Netherlands, 2018.

27. Abdelaty, A.M.; Azar, A.T.; Vaidyanathan, S.; Ouannas, A.; Radwan, A. Applications of Continuous-time Fractional Order Chaotic Systems. Math. Tech. Fract. Order Syst. 2018, 409-449. [CrossRef]

28. Soleimanizadeh, A.; Nekoui, M.A. Optimal type-2 fuzzy synchronization of two different fractional-order chaotic systems with variable orders with an application to secure communication. Soft Comput. 2021, 25, 6415-6426. [CrossRef]

29. Echenausía-Monroy, J.; Gilardi-Velázquez, H.; Jaimes-Reátegui, R.; Aboites, V.; Huerta-Cuellar, G. A physical interpretation of fractional-order-derivatives in a jerk system: Electronic approach. Commun. Nonlinear Sci. Numer. Simul. 2020, $90,105413$. [CrossRef]

30. Lai, Q.; Wan, Z.; Kuate, P.D.K.; Fotsin, H. Coexisting attractors, circuit implementation and synchronization control of a new chaotic system evolved from the simplest memristor chaotic circuit. Commun. Nonlinear Sci. Numer. Simul. 2020, 89, 105341. [CrossRef]

31. Rahman, Z.-A.S.A.; Al-Kashoash, H.A.A.; Ramadhan, S.M.; Al-Yasir, Y.I.A. Adaptive Control Synchronization of a Novel Memristive Chaotic System for Secure Communication Applications. Inventions 2019, 4, 30. [CrossRef]

32. Jasim, B.H.; Mjily, A.H.; AL-Aaragee, A.M.J. A novel 4 dimensional hyperchaotic system with its control, synchronization and implementation. Int. J. Electr. Comput. Eng. 2021, 11, 2974-2985.

33. Agrawal, S.; Srivastava, M.; Das, S. Synchronization of fractional order chaotic systems using active control method. Chaos Solitons Fractals 2012, 45, 737-752. [CrossRef]

34. Tavazoei, M.S.; Haeri, M. Synchronization of chaotic fractional-order systems via active sliding mode controller. Phys. A Stat. Mech. Appl. 2008, 387, 57-70. [CrossRef]

35. Kumar, S.; Matouk, A.E.; Chaudhary, H.; Kant, S. Control and synchronization of fractional-order chaotic satellite systems using feedback and adaptive control techniques. Int. J. Adapt. Control. Signal Process. 2021, 35, 484-497. [CrossRef]

36. Liu, L.; Du, C.; Zhang, X.; Li, J.; Shi, S. Adaptive Synchronization Strategy between Two Autonomous Dissipative Chaotic Systems Using Fractional-Order Mittag-Leffler Stability. Entropy 2019, 21, 383. [CrossRef]

37. Megherbi, O.; Hamiche, H.; Djennoune, S.; Bettayeb, M. A new contribution for the impulsive synchronization of fractional-order discrete-time chaotic systems. Nonlinear Dyn. 2017, 90, 1519-1533. [CrossRef]

38. Ortigueira, M.D.; Machado, J.T.; Trujillo, J.J. Fractional derivatives and periodic functions. Int. J. Dyn. Control. 2017, 5, 72-78. [CrossRef]

39. Huseynov, I.T.; Ahmadova, A.; Fernandez, A.; Mahmudov, N.I. Explicit analytical solutions of incommensurate fractional differential equation systems. Appl. Math. Comput. 2021, 390, 125590. [CrossRef]

40. Wang, C.; Ding, Q. A New Two-Dimensional Map with Hidden Attractors. Entropy 2018, 20, 322. [CrossRef] [PubMed]

41. Jiang, Y.; Zhang, B. Comparative Study of Riemann-Liouville and Caputo Derivative Definitions in Time-Domain Analysis of Fractional-Order Capacitor. IEEE Trans. Circuits Syst. II Express Briefs 2020, 67, 2184-2188. [CrossRef]

42. Zheng, Z.; Zhao, W.; Dai, H. A new definition of fractional derivative. Int. J. Nonlinear Mech. 2019, 108, 1-6. [CrossRef]

43. He, S.; Sun, K.; Banerjee, S. Dynamical properties and complexity in fractional-order diffusionless Lorenz system. Eur. Phys. J. Plus 2016, 131, 1-12. [CrossRef]

44. Garrappa, R. Numerical Solution of Fractional Differential Equations: A Survey and a Software Tutorial. Mathematics 2018, 6, 16. [CrossRef] 
45. Qi, G.; Xu, L.; Yang, X. Energy mechanism analysis for chaotic dynamics of gyrostat system and simulation of displacement orbit using COMSOL. Appl. Math. Model. 2021, 92, 333-348. [CrossRef]

46. Stankevich, N.V.; Kuznetsov, A.P.; Seleznev, E.P. Chaos and hyperchaos arising from the destruction of multifrequency tori. Chaos Solitons Fractals 2021, 147, 110998. [CrossRef]

47. Xu, Y.; Wang, H.; Li, Y.; Pei, B. Image encryption based on synchronization of fractional chaotic systems. Commun. Nonlinear Sci. Numer. Simul. 2014, 19, 3735-3744. [CrossRef]

48. Fan, X.; Wang, Z. A Fuzzy Lyapunov Function Method to Stability Analysis of Fractional Order T-S Fuzzy Systems. IEEE Trans. Fuzzy Syst. 2021, PP, 1. [CrossRef]

49. Nowak, R.D. Nonlinear system identification. Circuits Syst. Signal Process 2002, 21, 109-122. [CrossRef]

50. Sánchez-López, C. An experimental synthesis methodology of fractional-order chaotic attractors. Nonlinear Dyn. 2020, 100, 3907-3923. [CrossRef]

51. Due, A. Core ARM. Arduino Due. Retrieved 2017, 9, 2019. 